\title{
RIEMANNIAN METRICS INDUCED BY TWO IMMERSIONS
}

\author{
M. DO CARMO AND M. DAJCZER
}

\begin{abstract}
We consider the situation where a Riemannian manifold $M^{n}$ can be isometrically immersed into spaces $N^{n+1}(c)$ and $N^{n+q}(\tilde{c})$ with constant curvatures $c<\tilde{c}, q \leq n-3$, and show that this implies the existence, at each point $p \in M$, of an umbilic subspace $U_{p} \subset T_{p} M$, for both immersions, with $\operatorname{dim} U_{p} \geq n-q$. In particular, if $M^{n}$ can be isometrically immersed as a hypersurface into two spaces of distinct constant curvatures, $M^{n}$ is conformally flat.
\end{abstract}

\section{Introduction.}

(1.1) An $n$-dimensional Riemannian manifold $M^{n}$ is conformally flat if, for each point $p \in M$, there exists a conformal diffeomorphism of a neighborhood of $p$ onto an open set of the euclidean space $R^{n}$. Let $x: M^{n} \rightarrow \bar{M}^{k}$ be an immersion of a differentiable manifold $M^{n}$ into a Riemannian manifold $\bar{M}^{k}$, and let $\alpha: T_{p} M \times$ $T_{p} M \rightarrow\left(T_{p} M\right)^{\perp}$ be the second fundamental form of $x$ at $p \in M$; here $\left(T_{p} M\right)^{\perp}$ is the orthogonal complement of $d x_{p}\left(T_{p} M\right)$ in $T_{x(p)} \bar{M}$. We say that $U_{p} \subset T_{p} M$ is an umbilic subspace of $x$ at $p$ if $\langle\alpha(X, Y), \xi\rangle=\lambda\langle X, Y\rangle, \lambda=$ const, for all $X \in U_{p}$, all $\xi \in\left(T_{p} M\right)^{\perp}$ and all $Y \in T_{p} M$, where $\langle$,$\rangle denotes both the Riemannian metric$ on $\bar{M}$ and the Riemannian metric on $M$ induced by $x$. We will denote by $\bar{M}^{k}(x)$ a $k$-dimensional Riemannian manifold with constant sectional curvature $c$. It is well known that if $n \geq 4$ and $x: M^{n} \rightarrow \bar{M}^{n+1}(c)$ is an immersion, the metric induced on $M^{n}$ by $x$ is conformally flat iff, for each $\in M$, there exists an umbilic subspace $U_{p} \subset T_{p} M$ with $\operatorname{dim} U_{q} \geq n-1$. We will prove the following local theorem.

(1.2) ThEOREM. Let $M^{n}$ be a Riemannian manifold. Assume that $M^{n}$ can be isometrically immersed in both $\bar{M}^{n+1}(c)$ and $\bar{M}^{n+1}(\tilde{c}), \tilde{c}>c, q \leq n-3$. Then, for each $p \in M$, there exists an umbilic subspace $U_{p} \subset T_{p} M$ of both immersions with $\operatorname{dim} U_{p} \geq n-q$.

(1.3) Corollary. Let $M^{n}, n \geq 4$, be a Riemannian manifold. Assume that $M^{n}$ can be isometrically immersed in both $\bar{M}^{n+1}(c)$ and $\bar{M}^{n+1}(\tilde{c}), \tilde{c} \neq c$. Then $M^{n}$ is conformally flat.

(1.4) REMARK. Corollary (1.3) is, in a certain sense, the strongest restriction that can be expected under its hypothesis. In fact, it can be shown [1] that if an immersion $x: M^{n} \rightarrow \bar{M}^{n+1}(\tilde{c})$ of a differentiable manifold $M^{n}$ induces on $M^{n}$ a general conformally flat Riemannian metric, then, with such a metric, $M^{n}$ can be isometrically immersed into a $\bar{M}^{n+1}(c)$, for any $c<\tilde{c}$.

Received by the editors December 1, 1980.

1980 Mathematics Subject Classification. Primary 53C25; Secondary 53A20.

Key words and phrases. Isometric immersions, umbilic subspaces, conformally flat Riemannian manifolds. 
(1.5) REMARK. The bound on the dimension of $U_{p}$ given by Theorem (1.2) is sharp as shown by the following example. Let $n>3$ and let $M^{n}=S_{1}^{n-2} \times R^{2}$ be the Riemannian product of the sphere $S_{1}^{n-2}$ of curvature one with the euclidean space $R^{2}$. As the first immersion $x_{1}: M^{n} \rightarrow R^{n+1}$, take the product immersion of the canonical embedding $i_{n-1}: S_{1}^{n-2} \subset R^{n-1}$ with the identity map $R^{2} \subset$ $R^{2}$. To define the second immersion, consider the map $f: R^{2} \rightarrow R^{4}$ obtained by composing the immersion of $R^{2}$ as a flat torus in $S_{1}^{3}$ with $i_{4}: S_{1}^{3} \subset R^{4}$. By taking the product immersion $i_{n-1} \times f: S_{1}^{n-2} \times R^{2} \rightarrow R^{n+3}$, it is easily checked that $i_{n-1} \times f\left(S^{n-2} \times R^{3}\right)$ is contained in a sphere of radius, say, $1 / \sqrt{\tilde{c}}$, of $R^{n+3}$. This gives an immersion $x_{2}: M^{n} \rightarrow S_{\tilde{c}}^{n+2}$, and clearly there exists, for all $p \in M$, an umbilic subspace $U_{p}=T_{p}\left(S_{1}^{n-2}\right)$ of both immersions, with $\operatorname{dim} U_{p}=n-2$.

(1.6) REMARK. As the proof of Theorem (1.2) will show, we actually obtain that $\operatorname{dim} U_{p} \geq n-l$, where $l \leq q$ is the dimension of the first normal space at $p$ of the second immersion.

By imposing further restrictions on the immersions of Corollary (1.3) we can characterize those Riemannian metrics that will arise there.

(1.7) Corlllary. Let $M^{n}$ be as in the hypothesis of Corollary (1.3). Assume further that the first immersion has constant mean curvature $H$ and that the second immersion has constant mean curvature $\tilde{H}$. Then $M^{n}$ is either a space of constant curvature $M^{n}(a)$ or a Riemannian product $M^{n-1}(a) \times R$.

PROOF OF (1.7). Let $e_{1}, \ldots, e_{n}$ be an orthonormal basis of $T_{p} M$ such that $e_{1}, \ldots, e_{n-1}$ is a basis for the umbilic subspace $U_{p} \subset T_{p} M$ of both immersions given by Theorem (1.2). Relative to such a basis, let $\lambda, \mu$ (resp. $\beta, \gamma$ ) be the eigenvalues of the second fundamental form of the first (resp. second) immersion. From Gauss' equations, we easily see that the fact that $H$ and $\tilde{H}$ are constant implies that $\lambda$ and $\mu$ are also constant. The result follows from Ryan [3, p. 373].

2. Proof of Theorem (1.2).

(2.1) We will use the theory of flat bilinear forms as developed by J. D. Moore in [2, pp. 459-465]. We recall that, given vector spaces $V, W$, a $W$-valued bilinear form $\beta: V \times V \rightarrow W$ is flat relative to a real inner product (, ):W $W W \rightarrow R$ if

$$
(\beta(x, z), \beta(y, w))-(\beta(x, w), \beta(y, z))=0,
$$

for all $x, y, z, w \in V$. The nullity $N(\beta)$ of $\beta$ is

$$
N(\beta)=\{n \in V ; \beta(x, n)=0 \text {, for all } x \in V\},
$$

and $\beta$ is null if

$$
(\beta(x, y), \beta(z, w))=0, \quad \text { all } x, y, z, w \in V .
$$

Now let $x_{1}: M^{n} \rightarrow \bar{M}^{n+1}(c)$ and $x_{2}: M^{n} \rightarrow \bar{M}^{n+q}(\tilde{c})$ be the two immersions referred to in the statement, and denote by $\langle\rangle,,\langle,\rangle_{1},\langle,\rangle_{2}$ the Riemannian inner products of $M^{n}, \bar{M}^{n+1}(c), \bar{M}^{n+q}(\tilde{c})$, respectively. Fix throughout the proof a point $p \in M$ and let

$$
\alpha_{1}: T_{p} M \times T_{p} M \rightarrow\left(T_{p}(M)_{1}^{\perp}, \quad \alpha_{2}: T_{p} M \times T_{p} M \rightarrow\left(T_{p} M\right)_{\frac{1}{2}}^{\perp}\right.
$$

be the second fundamental forms of $x_{1}, x_{2}$, respectively. Let $N_{i}$ be the first normal space of $x_{i}$, i.e.,

$$
N_{i}=\operatorname{span}\left\{\eta \in\left(T_{p} M\right)_{i}^{\perp} ; \eta=\alpha_{i}(X, Y), X, Y \in T_{p} M\right\}, \quad i=1,2 .
$$


Set $W=N_{1} \oplus R \oplus N_{2}$, and define a Lorentzian inner product (, ) in $W$ by requiring that $()=,-\langle,\rangle_{1}$ in $N_{1},()=,\langle,\rangle_{2}$ in $N_{2}$, and that the direct summands of $W$ are pairwise orthogonal. Define a bilinear form $\beta: T_{p} M \times T_{p} M \rightarrow W$ by

$$
\beta(X, Y)=\alpha_{1}(X, Y)+\sqrt{\tilde{c}-c}\langle X, Y\rangle_{S}+\alpha_{2}(X, Y), \quad X, Y \in T_{p} M,
$$

where $\eta$ is a generator of $R$ with $(\eta, \eta)=1$.

It follows from Gauss' equations for $x_{1}$ and $x_{2}$ that $\beta$ is flat and, since $\tilde{c}-c>0$, $N(\beta)=0$. Notice that $\alpha_{1}$ is not zero; otherwise, the inner product (, ) in $W$ is positive definite, hence (Moore [2, p. 463, Corollary 1])

$$
0=\operatorname{dim} N(\beta) \geq \operatorname{dim} V-\operatorname{dim} W \geq n-(q+2) \geq 1,
$$

which is a contradiction.

Let $N$ be a vector that generates $N_{1}$ with $\langle N, N\rangle_{1}=-(N, N)=1$.

Assertion. There exists a unit vector $\eta_{0} \in R \oplus N_{2}$ such that

$$
\left(\alpha_{1}(X, Y), N\right)=\left(\sqrt{\tilde{c}-c}(X, Y\rangle_{\zeta}+\alpha_{2}(X, Y), \eta_{0}\right) .
$$

To prove the assertion, we use the fact that (Moore [2, p. 464, Corollary 3]) $W$ has a direct sum decomposition $W=W_{1} \oplus W_{2}$ such that the restrictions of $($,$) to$ $W_{1}$ and $W_{2}$ are nondegenerate, and if $\beta_{1}$ and $\beta_{2}$ are the $W_{1}$ - and $W_{2}$-components of $\beta$, respectively, then $\beta_{1}$ is null and $\operatorname{dim} N\left(\beta_{2}\right) \geq \operatorname{dim} T_{p} M-\operatorname{dim} W_{2}$.

It follows that $\beta_{1}$ is not zero; otherwise, $\beta=\beta_{2}$ and

$$
0=\operatorname{dim} N(\beta)=\operatorname{dim} N\left(\beta_{2}\right) \geq n-1 \text {. }
$$

Since $\beta_{1}$ is null, the restriction of (, ) to $W_{1}$ is Lorentzian. Thus $\operatorname{dim} W_{1} \geq 2$, and we can choose bases $e_{1}, \ldots, e_{k}$ of $W_{1}$ and $\delta_{0}, \ldots, \delta_{l+1}$ of $R \oplus N_{2}$, such that

$$
e_{1}=\cosh \varphi N+\sinh \varphi \delta_{1}, \quad e_{2}=\delta_{2}, \ldots, e_{k}=\delta_{k} .
$$

Thus, by writing

$$
\beta_{1}(X, Y)=-\left(\beta(X, Y), e_{1}\right) e_{1}+\sum_{\alpha=2}^{k}\left(\beta(X, Y), e_{\alpha}\right) e_{\alpha}
$$

we obtain that the condition for $\beta_{1}$ to be null is

$$
\left(\beta(X, Y), e_{1}\right)\left(\beta(Z, W), e_{1}\right)=\sum_{\alpha=2}^{k}\left(\beta(X, Y), e_{\alpha}\left(\beta(Z, W), e_{\alpha}\right),\right.
$$

$X, Y, Z, W \in T_{p} M$. Define linear maps $B$ and $D_{\alpha}$ of $T_{p} M, \alpha=2, \ldots, k$, by

$$
\langle B X, Y\rangle=\left(\beta(X, Y), e_{1}\right), \quad\left\langle D_{\alpha} X, Y\right\rangle=\left(\beta(X, Y), e_{\alpha}\right) .
$$

Then (2.3) is equivalent to

$$
\langle B X, Y\rangle\langle B Z, W\rangle=\sum_{\alpha}\left\langle D_{\alpha} X, Y\right\rangle\left\langle D_{\alpha} Z, W\right\rangle .
$$

We need the following lemma of linear algebra.

(2.5) LEMMA. Let $V$ be a finite-dimensional real vector space with a positive definite inner product $\langle$,$\rangle and let B$ and $D_{\alpha}, \alpha=2, \ldots, k$, be selfadjoint linear maps of $V$ such that (2.4) holds for all $X, Y, Z, W \in V$. Then there exist real numbers $c_{\alpha}$ with $\sum_{\alpha} c_{\alpha}^{2}=1$ such that $B=\sum_{\alpha} c_{\alpha} D_{\alpha}$. 
PROOF OF THE LEMMA. Let $Z_{1}, \ldots, Z_{n}, n=\operatorname{dim} V$, be a basis of $V$ that diagonalizes $B$, i.e., $\left\langle B Z_{i}, Z_{j}\right\rangle=\lambda_{i} \delta_{i j}, i, j=1, \ldots, n$. Then by (2.4)

$$
\left\langle B Z_{i}, Z_{j}\right\rangle^{2}=\sum_{\alpha}\left\langle D_{\alpha} Z_{i}, Z_{j}\right\rangle^{2} .
$$

It follows that $Z_{1}, \ldots, Z_{n}$ diagonalizes each $D_{\alpha}$. Define $\gamma_{i}^{\alpha}$ by $D_{\alpha} Z_{i}=\gamma_{i}^{\alpha} Z_{i}$. Then, again by (2.4),

$$
\lambda_{i} \lambda_{j}=\sum_{\alpha} \gamma_{i}^{\alpha} \gamma_{j}^{\alpha}
$$

We first notice form (2.6) that if some $\lambda_{i}=0$ then $\gamma_{i}^{\alpha}=0$ for all $\alpha$. Next, set $\gamma_{i}=\left(\gamma_{i}^{1}, \ldots, \gamma_{i}^{k}\right) \in R^{k}$ and notice that (3.6) means that, in the usual inner product - of $R^{k}$,

It follows that

$$
\left|\gamma_{i} \cdot \gamma_{j}\right|^{2}=\left\|\gamma_{i}\right\|^{2}\left\|\gamma_{j}\right\|^{2}
$$

$$
\frac{\gamma_{i}^{\alpha}}{\gamma_{j}^{\alpha}}=\frac{\gamma_{i}^{\alpha} \gamma_{j}^{\alpha}}{\left(\gamma_{j}^{\alpha}\right)^{2}}=\frac{\gamma_{i}^{\beta} \gamma_{j}^{\beta}}{\left(\gamma_{j}^{\beta}\right)^{2}}=\frac{\sum_{\alpha} \gamma_{i}^{\alpha} \gamma_{j}^{\alpha}}{\sum_{\alpha}\left(\gamma_{j}^{\alpha}\right)^{2}}=\frac{\lambda_{i}}{\lambda_{j}}
$$

hence, by setting $\gamma_{i}^{\alpha} / \lambda_{i}=\gamma_{j}^{\alpha} / \lambda_{j}=c_{\alpha}$, we obtain from (2.6) that $\sum_{\alpha} c_{\alpha}^{2}=1$. Finally, since $\left(c_{\alpha} \gamma_{i}^{\alpha}\right) / \lambda_{i}=c_{\alpha}^{2}$, we obtain that $\lambda_{i}=\sum_{\alpha} c_{\alpha} \gamma_{i}^{\alpha}$ and this proves Lemma (2.5).

(2.7) To complete the proof of the assertion, we set for convenience

$$
\bar{\alpha}(X, Y)=\sqrt{\tilde{c}-c}(X, Y\rangle_{\zeta}+\alpha_{2}(X, Y),
$$

and notice that Lemma (2.5) implies that

$$
-\cosh \varphi\left(\alpha_{1}(X, Y), N\right)+\sinh \varphi\left(\bar{\alpha}(X, Y), \delta_{1}\right)=\sum_{\alpha=2}^{k} c_{\alpha}\left(\bar{\alpha}(X, Y), \delta_{\alpha}\right)
$$

Thus

$$
\left(\alpha_{1}(X, Y), N\right)=\left(\bar{\alpha}(X, Y), \frac{\sum_{\alpha=2}^{k} c_{\alpha} \delta_{\alpha}-\sinh \varphi \delta_{1}}{-\cosh \varphi}\right)=\left(\bar{\alpha}(X, Y), \eta_{0}\right)
$$

where

$$
\eta_{0}=\frac{\sinh \varphi \delta_{1}-\sum_{\alpha=2}^{k} c_{\alpha} \delta_{\alpha}}{\cosh \varphi}
$$

is easily seen to have norm one. This proves the assertion.

(2.8) We now complete the proof of Theorem (1.2). Choose an orthonormal basis $\bar{\eta}_{1}, \eta_{2}, \ldots, \eta_{l}$ of $N_{2}$ so that

$$
\eta_{0}=\sin \theta \eta+\cos \theta \bar{\eta}_{1}
$$

Let $\eta_{1}=\cos \theta \eta-\sin \theta \bar{\eta}_{1}$ and set

$$
R^{l}=\operatorname{span}\left\{\eta_{1}, \ldots, \eta_{l}\right\} \text {. }
$$

Define a bilinear form $\gamma: T_{p} M \times T_{p} M \rightarrow R^{l}$ by

$$
\gamma(X, Y)=\left(\sqrt{\tilde{c}-c}\langle X, Y\rangle \eta+\alpha_{2}(X, Y), \eta_{1}\right) \eta_{1}+\sum_{j=2}^{l}\left\langle\alpha_{2}(X, Y), \eta_{j}\right\rangle_{2} \eta_{j}
$$


By Gauss' equations for $x_{1}$ and $x_{2}, \gamma$ is flat relative to the (Riemannian) inner product obtained by restricting $($,$) to R^{l}$. It follows from Moore $([2$, p. 463, Corollary 1]) thadim $N(\gamma) \geq n-l \geq n-q$. On the other hand, $X \in N(\gamma)$ if and only if, for all $Y \in T_{p} M$, both conditions below are satisfied:

$$
\left\{\begin{array}{l}
\text { (i) } \quad \cos \theta \sqrt{\tilde{c}-c}\langle X, Y\rangle-\sin \theta\left\langle\alpha_{2}(X, Y), \bar{\eta}_{1}\right\rangle_{2}=0, \\
\text { (ii) }\left\langle\alpha_{2}(X, Y), \eta_{j}\right\rangle_{2}=0, \quad j \geq 2 .
\end{array}\right.
$$

Notice that by (2.2) and (2.9)

$$
\left(\alpha_{1}(X, Y), N\right)=\sqrt{\tilde{c}-c}(X, Y\rangle \sin \theta+\left\langle\alpha_{2}(X, Y), \bar{\eta}_{1}\right\rangle_{2} \cos \theta .
$$

It follows by (2.10) (i) that $\sin \theta \neq 0$, and by (2.11) that we can assume that $\cos \theta \neq 0$ (otherwise the whole $T_{p} M$ is an umbilic subspace). Thus $X \in N(\gamma)$ if and only if, for all $Y \in T_{p} M$,

$$
\alpha_{2}(X, Y)=\operatorname{cotg} \theta \sqrt{\tilde{c}-c}(X, Y) \bar{\eta}_{1},
$$

and by (2.11) this is equivalent to

$$
\alpha_{1}(X, Y)=\frac{\sqrt{\tilde{c}-c}}{\cos \theta}\langle X, Y\rangle
$$

Therefore, $N(\gamma) \subset T_{p} M$ is an umbilic subspace of both $x_{1}$ and $x_{2}$ with $\operatorname{dim} N(\gamma) \geq n-q$. This proves Theorem (1.2).

\section{REFERENCES}

1. M. do Carmo and M. Dajczer, General conformally flat hypersurfaces (preprint).

2. J. D. Moore, Submanifolds of constant positive curvature. I, Duke Math. J. 44 (1977), $449-484$.

3. P. Ryan, Homogeneity and some curvature conditions for hypersurfaces, Tôhoku Math. J. 21 (1969), 363-388.

Instituto de Matemática Pura e Aplicada (IMPA), Estrada Dona Castorina, 110, CEP 22.460, RIO DE JANEIRO, RJ, BRAZIL 